\title{
CRO based Single Phase Parameter Measurement of Electrical Supply
}

\author{
Charu Maggu \\ Lecturer, Electrical SBBSIET, Punjab, India
}

\author{
S.K Pahuja, PhD \\ Professor, ICE, NITJ, Punjab, India
}

\begin{abstract}
Present paper proposes a power measurement method of a single phase electrical load (RLC). Real time monitoring of power supply is essential for its continuous and reliable operation. So, this paper also presents a low cost, low power consuming system that can be used for fast and precise domestic power supply parameter monitoring. Simulations analysis of the voltage waveform, current waveform and the phase difference between these two signals were done using CRO. By using this measurement techniques behavior of any electrical appliances can be checked and improvement in its working can be possible after that in it. And also using this measurement technique we can check which types of harmonics are flowing in the appliances during its normal working condition.
\end{abstract}

\section{Keywords}

Power measurement, transjector, voltage divider, phase difference.

\section{INTRODUCTION}

The requirement of the load power measurements is repetitive in the electrical engineering labs and installations. There are equipment's available in the market that can be used to measure these quantities. Accurate measurement of power and other AC quantities is very vital at all levels of the electrical power system,. The objective of this paper is to design power measurement system of an electrical load. The load to be considered in the present study is RLC parallel circuit. Measurement of single phase instantaneous voltage and current has been measured with help of CRO. Voltage is measured with the help of voltage divider and the instantaneous current is measured with the help transjector. Then these waveforms are observed on the screen of CRO. The proposed method is a low cost power measurement technique. The load taken into consideration is resistive load, inductive load and capactive load. One signal transducted via transjector and the other signal transducted through the voltage divider. Firstly main supply voltage which is nearly equal to $230 \mathrm{~V}$ is applied to voltage divider which steps down this voltage to $1 / 100$ part of applied voltage as a result for $230 \mathrm{~V}$ corresponding $2.3 \mathrm{~V}$ is available at the output terminal of voltage divider. Voltage divider is consist of two part first one is $99 \mathrm{~K} \Omega$ and other part is $1 \mathrm{~K} \Omega$.total voltage is applied across $100 \mathrm{~K} \Omega$ and output voltage across $1 \mathrm{~K} \Omega$ is taken out from the divider Voltage from $1 \mathrm{~K} \Omega$ is then connected to the channel 1 of CRO.As a result waveform is start plotting on the screen of CRO. Output voltage corresponding to supply voltage is then can be easily calculated from the division easily. Now for the measurement of current in circuit can also be measured through CRO. To measure current which is flowing through RLC load connect $1 \Omega$ resistance parallel to neutral wire. As a result this current is converted in voltage signal. This signal is now connect to channel 2 of CRO.

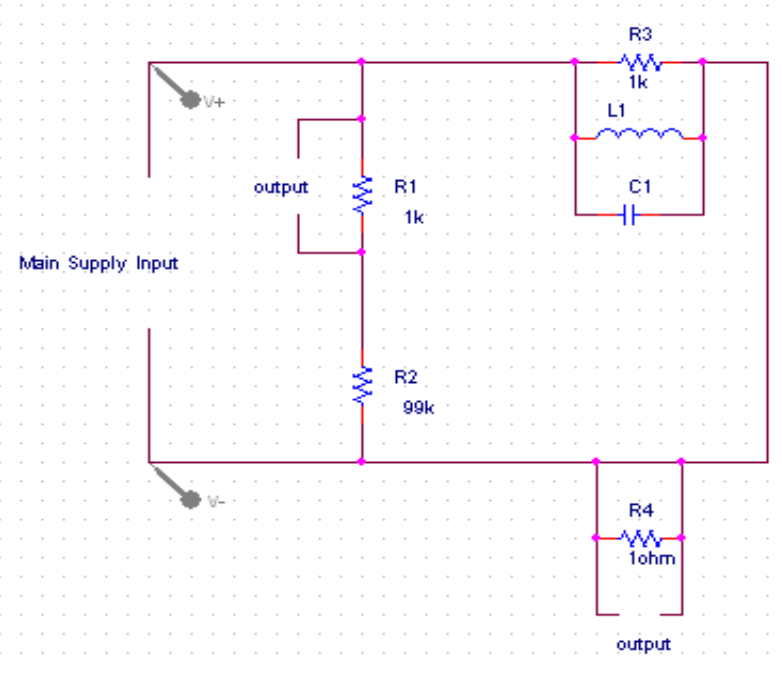

Figure 1 single line diagram of circuit

\section{INTRODUCTION TO OSCILLOSCOPE}

An oscilloscope is a device for recording and visualizing the instantaneous value of alternating voltage, current and is used in many professions and educational institutions. Oscilloscopes are well documented, but only to study the theory is not sufficient to learn how to use these measuring instruments [1].

\subsection{What is an Oscilloscope}

Oscilloscope is a computing device which can show several waveforms \& voltages, Frequency, time period, wavelength of any signal can be measured using Oscilloscope. The oscilloscope is mainly a graph-displaying device - it draws a graph of an electrical signal. In most applications the graph depicts how signals change over time. An oscilloscope is a laboratory instrument normally used to display and examine the waveform of electronic signals. In effect, the device draws a graph of the instantaneous signal voltage as a function of time. An oscilloscope can display alternating current (AC) or pulsating direct current (DC) waveforms having a frequency as low as almost 1 hertz $(\mathrm{Hz})$ or as high as several megahertz (MHz)

\subsection{How does An Oscilloscope Work?}

A typical oscilloscope works by measuring the voltage signal as it travels through the object. It is used to help find breaks in the circuits or shorts. A traditional oscilloscope works in almost exactly the same way as a traditional (cathode-ray tube) television. In an oscilloscope, the electron beams work the same way but instead of building up a picture they draw a graph. When you watch a line being drawn on an oscilloscope screen, what you're actually 
The time/division switch is set to $0.5 \mathrm{~ms} /$ division looking at is an electron beam wobbling up and down. The electrical signals feeding into the $\mathrm{x}$ and $\mathrm{y}$ connections well become the $\mathrm{x}$ and $\mathrm{y}$ values on your on-screen chart.

\subsection{How to Use an Oscilloscope?}

The only method to use oscilloscope is when dealing with electronics it will use to find faulty electrical circuits. Cathode ray oscilloscope is the original oscilloscope and uses a high potential difference. Electrons are pushed off a plate from one end using very high voltage and directed by electric fields to the phosphor screen that fluoresces when knockout by an electron.

\section{CATHODE-RAY OSCILLOSCOPE (CRO) OBJECTIVE}

- To familiarize the basic structure of a cathode-ray Oscilloscope.

- To get aware with the use of different control switches of the device.

- To envision an AC signal, measure the amplitude and the frequency.

The device comprises mainly of a vacuum tube which comprises a cathode; anode, grid, X\&Y-plates, and screen which is also known as fluorescent screen (see Figure below). When the cathode is heated (by applying a slight potential difference across its terminals), it radiates electrons. Having a potential difference across the cathode and the anode, accelerate the emitted electrons towards the anode, developing an electron beam, which passes to fall on the screen. When the firm electron beam strikes the fluorescent screen, a bright spot is produced. The grid, which is positioned between two electrodes, controls the intensity of electrons passing through it thereby controlling the intensity of the electron beam. The Y\&X plates produce deflecting electron beam horizontally and vertically [1].

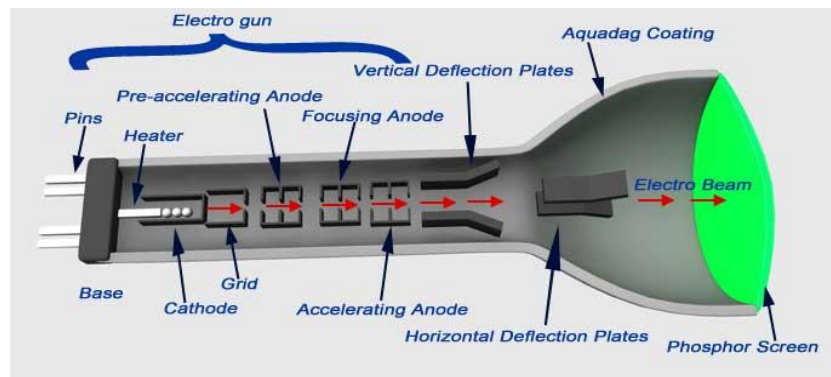

Figure 2 Internal structure of CRO

A sweep generator is coupled to the X-plates, which moves the bright spot horizontally across the screen and repeats that at a definite frequency as the source of the signal. The voltage to be calculated is applied to the Y-plates of the scope. The combined sweep and $\mathrm{Y}$ voltages create a graph presenting the variation of voltage with time, as shown in

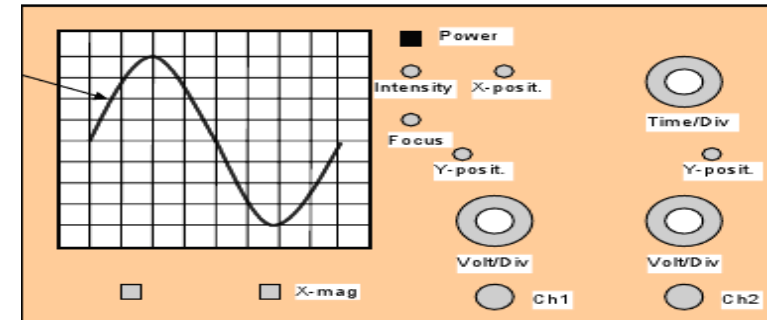

Fig.3. Front panel of the CRO

An oscilloscope is a basically type of electronic test instrument that permits observation of continuously varying signal voltages, usually as a 2-D graph of one or more electrical potential differences using the vertical or ' $\mathrm{Y}$ ' axis, plotted as a function of time along horizontal or ' $\mathrm{x}$ ' axis. Though scope displays voltage on its vertical axis, any other signal that can be transformed to a voltage can be displayed as well on screen. In most cases, oscilloscopes display events that repeat with either no variation, or varying slowly. Scopes are commonly used to observe the wave shape of an electrical signal. In addition to the amplitude of the observed signal, an oscilloscope can show distortion of observed signal, the time between events (such as pulse width, period, or rise time) and relative timing of 2 related signals. Each channel of scope is really just a high quality amplifier with low noise, high bandwidth and gain which connects to the vertical deflector of scope. Most oscilloscopes have a test point that generates a low frequency square wave and you can use it to setup the oscilloscope and its probes. First of all adjust the focus and intensity to get the best display - a nice sharp line. Then set the input to ac and plug in the probe to the channel you are looking at and then attach the probe tip to the test point. You should then see the square wave - adjust the channel amplifier until its a good size in the current systems are using electronic device. Two types are CRO are available, analog and digital CROs. They are using to measure various parameters. For this, many controls are available which are elaborate as shown below.

The controls option on a scope can be divided into four groups.

1 Hold/Control (on/off, brightness, focus)

2 Horizontal control (X position, $\mathrm{X}$ amplitude, time/division)

3 Vertical control (Y position, Y amplitude, Volts/division)

4 Trigger/synchronization (level, +/-, ac/dc, external)

Set any controls to "calibrate" for the correct position. Adjust the controls to obtain a trace on the screen. Adjust the vertical and horizontal controls to display a few cycles of a waveform. Adjust the trigger/synchronization controls to make the display stationary. It is best to display nearly two cycles and make them as large as possible in height. Measure the amplitude and periodic time of signal. Lets take an example to calculate the frequency of any signal 


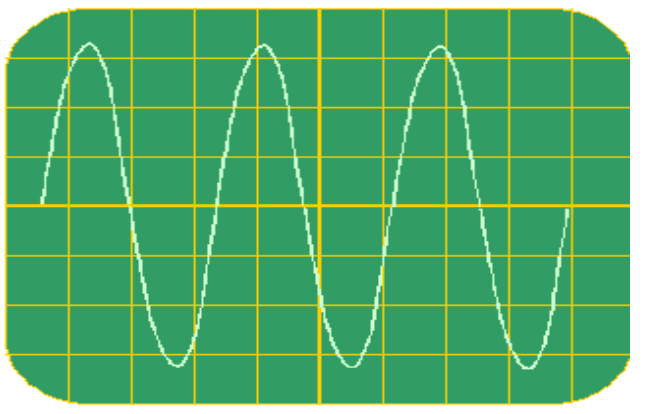

Figure 4 sine wave signal to calculate frequency

In the diagram, $\mathrm{Y}$ gain is set at $2 \mathrm{~V} / \mathrm{cm}$ and the total height of the wave from peak to trough is $6.4 \mathrm{~cm}$. Therefore

$\mathrm{V}$ peak to peak $=12.8 \mathrm{~V}$

$$
\mathrm{Vo}=6.4 \mathrm{~V}
$$

. The periodic time is $0.5 * 6.4=1.45 \times 10-3$

Frequency $=1 /$ periodic time $=690 \mathrm{~Hz}$.

Similarly voltage and current of the two signals can also be observed and their amplitude can easily be calculated without using any other conventional instruments [2-4].

\subsection{Frequency and Period}

Frequency is the number of happenings of a iterating event per unit time .It is also stated to as temporal frequency, If signal recurrences, it has a frequency. That frequency is measured in $\mathrm{Hz}$ (Hertz) and equals the number of times the signal repeats itself in a time of one second (the cycles per second). The period is the interval of time of one cycle in a repeating occurrence, thus the period is the reciprocal of the frequency. Repeating signal also has a period and this is the amount of time it takes by signal to complete one whole cycle. Frequency and period are specifically different, yet related quantities. Frequency also refers to how often something take place. Period refers to the time it takes something to happen. As a matter of convenience, longer and slower waves, such as ocean surface waves, tend to be designated by wave period rather than frequency. Short and fast waves, like audio and radio wave, are usually described by their frequency instead of period. Period and frequency are related to each other as a reciprocal, so that 1/period equals the frequency and $1 /$ frequency equals the period. So, for example, the sine wave has a frequency of $10 \mathrm{~Hz}$ and a period of $1 / 10$ second as shown in fig 5. Voltage is the amount of electric potential have between two points in a circuit. Usually one of these points is ground i.e. at zero volts but not always. We may want to measure the voltage from the maximum peak to the minimum peak of a waveform, denoted as the peak-topeak voltage. Period is a time quantity. Frequency is the cycles/second. Period is the seconds/cycle. Consider our wave with a period of 5 seconds. Since the wave completes one cycle every two seconds, then its frequency is one half or 0.2 $\mathrm{Hz}$. So you see - period and frequency are reciprocals of each other. We can characterize their relationship with a simple equation. Greater the period is for a wave, lesser wave cycles can fit within a second and as results lower the frequency of signal. Likewise, a wave with larger frequency would have to fit more wave cycles into each second, meaning the period of each cycle would have to be smaller. No matter what kind of wave we are looking at, the period and frequency inversely proportional to each other always.

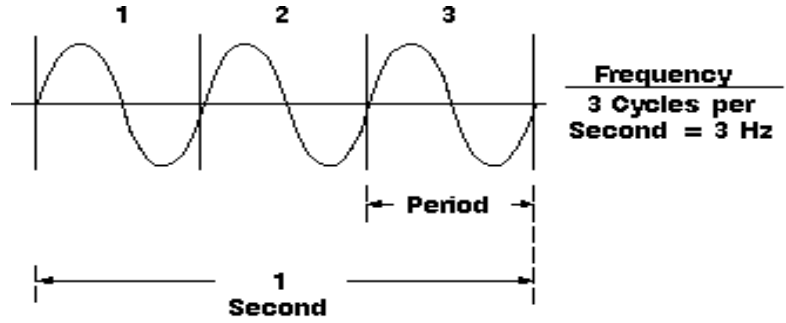

Figure 5 Relation between frequency and period.

\subsection{Current}

Current is the amount of flow of electrical charge carriers, generally electrons or electron-deficient atoms. One ampere of current signifies one coulomb of electrical charge $(6.24 \mathrm{x}$ 1018 charge carriers) moving past a specific point in one second. To measure the current of RLC circuit insert $1 \Omega$ resistance in the neutral wire. Due to this resistance drop occur across it because a neutral wire has some voltage as compare to phase wire. Then send this output signal of $1 \Omega$ resistance to the CRO and observe the voltage of this signal. Now divide this voltage drop with the resistance which is connected in the neutral wire. Result which we get after division is net current which is flowing in the circuit. The SI unit for measuring an electric current is ampere which is denoted by A. Since the current in a wire or component can flow in both direction, when a variable $I$ is defined to characterize that current, the direction representing positive current must be specified, generally by an arrow on the circuit schematic diagram.

\subsection{Phase}

Phase in sinusoidal functions or in waves has two different, but closely correlated meanings. One is the initial angle of a sinusoidal function at its origin and sometimes also called phase offset or phase difference of signal. Another usage is the portion of the wave cycle that has elapsed relative to the origin Phase is best explained by considering a sine wave. Sine waves are based on circular motion. One cycle of a sine wave has 360 degrees. Using degrees, we can refer to the phase angle of a sine wave when we want to describe how much of the period has elapsed. Frequently what is required in practice is a measurement of the phase difference among two signals of the same frequency; that is, it is require measuring the relative phase between two signals rather than the absolute phase of either one. Often, in the measurement of the relative phase between two signals, both signals are derived from the similar source. For example these signals might be the current and voltage of a power system; the relative phase, between the current and voltage would then be useful for observing power usage, since the latter is proportionate to the cosine of $\boldsymbol{\omega}$. Several methods are available for the measurement of "relative phase." One basic method involves forming "Lissajous figures" on an oscilloscope. In this method, first of all two signals of concern is fed into the vertical input of a $\mathrm{CRO}$ and the other is fed into the horizontal input. The result on the oscilloscope screen is an ellipse, the intercept and maximum height of which can be used to determine the relative phase [7].

\section{EXPERIMENTAL RESULTS}

In this experiment, firstly apply the main supply across the divider which consist of $100 \mathrm{k} \Omega$. Divide this resistance into two parts. First part consist of $1 \mathrm{k} \Omega$ and second part consist of $99 \mathrm{k} \Omega$.now take connect output wires across the $1 \mathrm{~K} \Omega$.For $230 \mathrm{~V}$ (mains supply) corresponding $2.3 \mathrm{~V}$ voltage is observed. 
Then observe this signal on the CRO and check the readings whether it is correct or not

\subsection{First case: Voltage measurement}

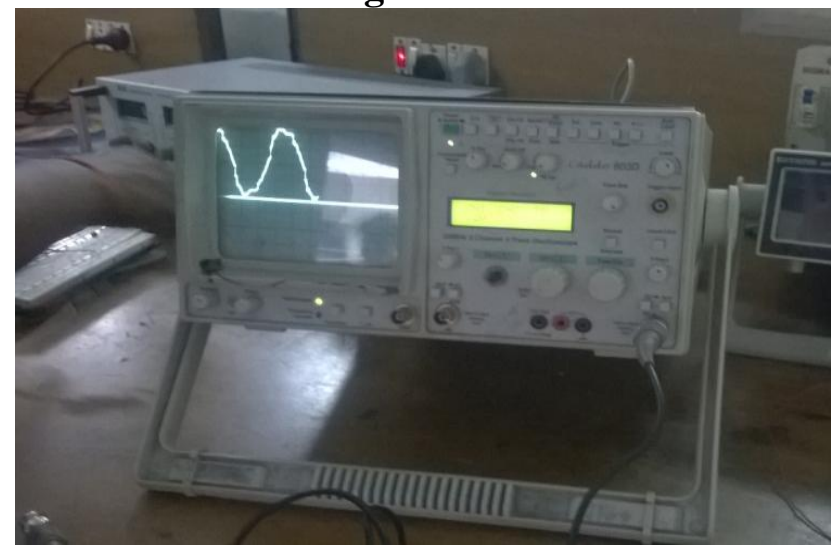

Figure 3.1: Instantaneous voltage waveform of main supply

Table 3.1: Comparison of voltage readings

\begin{tabular}{|l|l|l|l|l|}
\hline $\begin{array}{l}\text { Sr. } \\
\text { No. }\end{array}$ & $\begin{array}{l}\text { Reading } \\
\text { from } \\
\text { CRO }\end{array}$ & $\begin{array}{l}\text { Multiplying } \\
\text { factor }\end{array}$ & $\begin{array}{l}\text { Actual } \\
\text { voltage } \\
\text { read } \\
\text { through } \\
\text { CRO }\end{array}$ & $\begin{array}{l}\text { Values } \\
\text { obtained } \\
\text { from } \\
\text { voltmeter }\end{array}$ \\
\hline 1 & $2.29 \mathrm{~V}$ & 100 & $229 \mathrm{~V}$ & $230 \mathrm{~V}$ \\
\hline 2 & $2.25 \mathrm{~V}$ & 100 & $225 \mathrm{~V}$ & $227 \mathrm{~V}$ \\
\hline 3 & $2.28 \mathrm{~V}$ & 100 & $228 \mathrm{~V}$ & $228.5 \mathrm{~V}$ \\
\hline 4 & $2.2 \mathrm{~V}$ & 100 & $220 \mathrm{~V}$ & $221.5 \mathrm{~V}$ \\
\hline 5 & $2.3 \mathrm{~V}$ & 100 & $230 \mathrm{~V}$ & $230 \mathrm{~V}$ \\
\hline
\end{tabular}

\subsection{Second case: Current measurement}

In this experiment, for the determination of flowing current in the circuit connect $1 \Omega$ resistance wire in parallel to the neutral wire. As a result drop takes place across the wire due to the current flowing in the circuit. Now this voltage drop gives us the value of current of the circuit. The purpose of connecting this $1 \Omega$ shunt is to convert the current signal into voltage

Table 3.2: comparison of current readings

\begin{tabular}{|l|l|l|l|}
\hline $\begin{array}{l}\text { Sr. } \\
\text { No. }\end{array}$ & Load & $\begin{array}{l}\text { Current obtained } \\
\text { from CRO }\end{array}$ & $\begin{array}{l}\text { Values obtained } \\
\text { from Current }\end{array}$ \\
\hline 1 & RLC & $1.09 \mathrm{~A}$ & $1.12 \mathrm{~A}$ \\
\hline 2 & RL & $1.03 \mathrm{~A}$ & $1.18 \mathrm{~A}$ \\
\hline 3 & LC & $.331 \mathrm{~A}$ & $.33 \mathrm{~A}$ \\
\hline 4 & RC & $.8 \mathrm{~A}$ & $.91 \mathrm{~A}$ \\
\hline 5 & $\mathrm{R}$ & $.86 \mathrm{~A}$ & $.89 \mathrm{~A}$ \\
\hline
\end{tabular}

\begin{tabular}{|l|l|l|l|}
\hline 6 & $\mathrm{~L}$ & $.54 \mathrm{~A}$ & $.55 \mathrm{~A}$ \\
\hline 7 & $\mathrm{C}$ & $.155 \mathrm{~A}$ & $.20 \mathrm{~A}$ \\
\hline
\end{tabular}

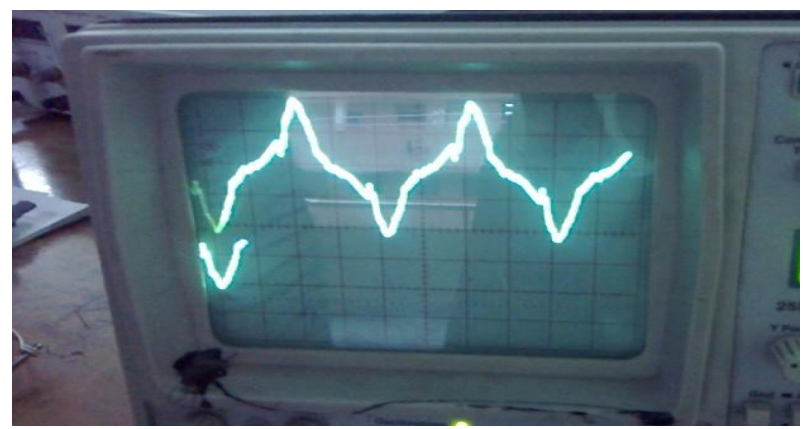

Fig 3.2: Instantaneous current waveform of main supply

\section{CONCLUSION AND FUTURE SCOPE}

In this project, disturbances in the two signal i.e. voltage and current is observed. How power factor is varied by changing the load can be easily studied. However, the setup is not tested for capacitive load. Further same set up can be use in conjunction with the DSP or Microcontroller and can be used for other supply parameter estimation specially the supply frequency, energy consumed and that too using a single measurement, that may be the part of future work and scope.

\section{REFERENCES}

[1] Kularatna,Nihal (2007), "Fundamentals of Oscilloscopes", Digital and Analogue Instrumentation: Testing and Measurement, Institution of Engineering and Technology, pp. 165-208, ISBN 978-0-85296-999-1

[2] Sawhney AK, Sawhney P (2003) Electrical and electronics measurements and measuring instruments. Measurement and Measurement Systems.

[3] Artigas JI, Urriza I, Acero J, Barragán LA, Navarro D, et al. (2009) Power Measurement by Output-Current Integration in Series Resonant Inverters. IEEE $\mathrm{T}$ Ind Electron 56: 559-567.

[4] Simpson RH (1998) Instrumentation, measurement techniques, and analytical tools in power quality studies. IEEE T Ind Appl 34: 534-548.

[5] Shinde Sachin, Prabhu Sapna,(2013)"Labview based digital CRO for electronic measurement techniques" International Journal of Engineering Research and Applications (IJERA) ISSN: 2248-9622, vol. 3, issue 1, pp.693-69

\section{AUTHOR PROFILE}

Charu Maggu B.tech in EE pursing M.tech in instrumentation and control from NITJ. Presently working as a lecturer in SBBSIET in EE department

Dr.S.K Pahuja 